FIAN/TD-03/09

ITEP/TH-06/09

IHES/P/09/06

\title{
On two-dimensional quantum gravity and quasiclassical integrable hierarchies *
}

\author{
A. Marshakov \\ Theory Department, P.N.Lebedev Physics Institute, \\ Institute of Theoretical and Experimental Physics, \\ Moscow, Russia \\ e-mail: mars@lpi.ru, mars@itep.ru
}

to the memory of

Alesha Zamolodchikov

The main results for the two-dimensional quantum gravity, conjectured from the matrix model or integrable approach, are presented in the form to be compared with the world-sheet or Liouville approach. In spherical limit the integrable side for minimal string theories is completely formulated using simple manipulations with two polynomials, based on residue formulas from quasiclassical hierarchies. Explicit computations for particular models are performed and certain delicate issues of nontrivial relations among them are discussed. They concern the connections between different theories, obtained as expansions of basically the same stringy solution to dispersionless KP hierarchy in different backgrounds, characterized by nonvanishing background values of different times, being the simplest known example of change of the quantum numbers of physical observables, when moving to a different point in the moduli space of the theory.

*based on talk presented at the conference Liouville field theory and statistical models, (dedicated to the memory of Alexei B. Zamolodchikov), Moscow, June 2008 


\section{Introduction}

The problem of solving two-dimensional quantum gravity exists already more than twenty, or even more than twenty-five years. By its basic definition one usually takes the Polyakov path integral [1], where the integration over the metrics on two-dimensional string world-sheets has been reduced to study of naively simple, but in fact quite nontrivial two-dimensional conformal Liouville field theory. The world-sheet approach allowed to determine immediately only the relatively simple quantities - like scaling dimensions - of the operators of the two-dimensional quantum gravity $[2,3]$. The computation of their correlators - even on the world-sheets of simplest spherical topology - appeared to be the problem of much higher complexity, and was (yet only partially) solved very recently.

Fortunately, the two-dimensional quantum gravity is a renormalizable theory - in the most physically important sense of the word, which means that the details of regularization of the theory at microscopic scale do not affect its macroscopic properties: the "observable" scaling dimensions and correlators. In different words, two dimensional quantum gravity possesses strong universality property - meaning that quite different methods of the computation gives rise basically to the same result.

The first sign of this was observed already in the middle of 80-s of the last century. The idea of summing over the discrete triangulations of world sheets instead of the integrations over the metric in continuous theory had demonstrated its efficiency in two-dimensional case, quite in contrast with the nonrenormalizable gravity of higher dimensions. Moreover, it turned out that summing over triangulations of the two-dimensional surfaces can be itself reformulated as summing over the fat graphs of the matrix models [4]. The duality between matrix model (the zero-dimensional gauge theory) and continuous two-dimensional world-sheet gravity is in fact nothing but the first studied example of the famous nowadays gauge/string duality.

By the matrix model approach two-dimensional quantum gravity was claimed to be "completely solved" [5] in the beginning of 90 -s of the previous century. This solution was nicely formulated $[6,7]$ in terms of special stringy solutions to the hierarchies of integrable equations, being all the well-known polynomial reductions of the Kadomtsev-Petviashvili (KP) hierarchy. In practice, this has opened a possibility to compute exactly the correlators in two-dimensional gravity (in the framework of "matrix model" approach) at least in the spherical approximation (when all closed string loops are suppressed) by methods of dispersionless KP hierarchy, which turn this problem into the problem of solving algebraic nonlinear equations. Below, following [8], we shall demonstrate how this way leads straightforwardly to the computation of invariant correlation numbers - the ratios of the correlation functions which do not depend upon normalizations of particular operators.

However, it is still a great puzzle and, at least partially, an open problem, whether the matrix model approach leads exactly to the same results as the original world-sheet approach. Partially this is related to the fact, that the world-sheet quantum Liouville theory of [1] is 
a rather specific two-dimensional quantum field theory, which is yet to be fully understood. The two- and three- point functions in Liouville theory were computed in early 90-s [9, 10], but it turned out, that only after discovery of the higher order equations of motion by Alesha Zamolodchikov [11], it appeared to be possible to compute the generic multipoint correlation functions of the operators of minimal $(p, q)$ models coupled to the two-dimensional Liouville gravity, where the integrands on the moduli spaces of world-sheets with punctures are basically reduced after using the higher order equations to the total derivatives.

These correlation functions could be now compared with the results extracted from the "matrix model" approach, or more strictly, from the formulation of minimal string theory in the language of integrable hierarchies.

\section{2 dKP for (p,q)-critical points}

According to widely beleived hypothesis, the so called $(p, q)$ critical points of two-dimensional gravity (or $(p, q)$ minimal string theory) are most effectively described by the tau-function of $p$-reduced KP hierarchy, satisfying string equation. The logarithm of this tau-function should be further expanded around certain background values of the time-variables, with necessary $t_{p+q} \neq 0$. In particular, it means that the correlators on world-sheets of spherical topology (the only ones, partially computed by now by means of two-dimensional conformal field theory $[11,12])$ are governed by quasiclassical tau-function of dispersionless KP or dKP hierarchy, which is a very reduced case of generic quasiclassical hierarchy from [13].

For each $(p, q)$-th minimal theory one should consider a solution of the $p$-reduced dKP hierarchy, or more strictly, its expansion in the vicinity of nonvanishing $t_{p+q}=\frac{p}{p+q}$ and vanishing other times, perhaps except for cosmological constant $x$, chosen in a different way for the different theories (the so called conformal backgrounds). If $q=p+1$ (the unitary series) the cosmological constants $x \sim t_{1}$ basically coincides with the main first time of the KP hierarchy, but for "non-unitary" backgrounds the quantum numbers change, and this causes certain nontrivial relations on the space of KP solutions to be discussed below.

\section{$2.1 \quad$ Residue formulas}

The geometric formulation of results for minimal string theories in terms of the quasiclassical hierarchy can be sketched in the following way:

- For each $(p, q)$-th point take a pair of polynomials

$$
\begin{aligned}
& X=\lambda^{p}+\ldots \\
& Y=\lambda^{q}+\ldots
\end{aligned}
$$


of degrees $p$ and $q$ respectively. They can be thought of as a dispersionless version of the Lax and Orlov-Shulman operators of KP theory

$$
\begin{gathered}
{[\hat{X}, \hat{Y}]=\hbar} \\
\hat{X}=\partial^{p}+\ldots, \quad \hat{Y}=\partial^{q}+\ldots
\end{gathered}
$$

or as a pair of (here already integrated) Krichever differentials with the fixed periods on a complex curve (for dKP - a rational curve with global uniformizing parameter $\lambda$ ). It is also convenient to combine these polynomials into a generating differential

$$
d S=Y d X
$$

whose periods and singularities define the variables of the quasiclassical hierarchy. Since on rational curve $\left(\lambda\right.$-plane or Riemann sphere with the marked point $P_{0}$, where $\lambda=\infty$ ) all periods of (3) vanish, the time variables are related with the residues or singular part of expansion of the differential $d S$ at the point $P_{0}$.

- The variables of dispersionless KP hierarchy are therefore introduced by residue formulas $[13,14,15]$

$$
\begin{gathered}
t_{k}=\frac{1}{k} \operatorname{res}_{P_{0}} \xi^{-k} d S, \quad k>0 \\
\frac{\partial \mathcal{F}}{\partial t_{k}}=\operatorname{res}_{P_{0}} \xi^{k} d S, \quad k>0
\end{gathered}
$$

where

$$
\xi=X^{\frac{1}{p}}=\lambda\left(1+\ldots+\frac{X_{0}}{\lambda^{p}}\right)^{\frac{1}{p}}
$$

is the distinguished inverse local co-ordinate at the point $P_{0}$, where $\lambda\left(P_{0}\right)=\infty$ and $\xi\left(P_{0}\right)=\infty$. From (4) it also follows for the second derivatives

$$
\frac{\partial^{2} \mathcal{F}}{\partial t_{n} \partial t_{k}}=\operatorname{res}_{P_{0}}\left(\xi^{k} d H_{n}\right)
$$

while the third derivatives are given by the formula

$$
\frac{\partial^{3} \mathcal{F}}{\partial t_{k} \partial t_{l} \partial t_{n}}=\operatorname{res}_{d X=0}\left(\frac{d H_{k} d H_{l} d H_{n}}{d X d Y}\right)
$$

In (6) and (7) the set of one-forms

$$
d H_{k}=\frac{\partial d S}{\partial t_{k}}, \quad k \geq 1
$$

(derivatives are taken at fixed $X$ ) corresponds to dispersionless limit of KP flows and can be integrated up to polynomial expressions

$$
H_{k}=X(\lambda)_{+}^{k / p}
$$

in uniformizing co-ordinate $\lambda=H_{1}$. 
Note also, that the tau-functions of $(p, q)$ and $(q, p)$ theories do not coincide, but are related by the Legendre or Fourier transform [16], exchanging the polynomials (1) by each other $X \leftrightarrow Y$.

\subsection{Solution to dKP}

The fact, that one-forms (8) can be integrated up to the polynomials (9) leads to explicit expression for the integrated generating differential (3), or

$$
S=\sum_{k=1}^{p+q} t_{k} H_{k}=\sum_{k=1}^{p+q} t_{k} X^{k / p}(\lambda)_{+}, \quad k \bmod p
$$

depending already upon the coefficients of the polynomial $X(\lambda)$ only. In different words, formula (10) means, that the first part of the equations (4) has been already effectively resolved for the coefficients of $Y(\lambda)$. The dependence of coefficients of $X(\lambda)=\lambda^{p}+\sum_{k=0}^{p-2} X_{k} \lambda^{k}$ over the KP times (4) is determined in the most easy way from $\left.d S\right|_{d X=0}=0$, which is now a system of $p-1$ "hodograph" equations $\frac{d S}{d \lambda}=0$ imposed at $p-1$ roots of $X^{\prime}(\lambda)=0$.

A simple observation, that any hamiltonian (9) is a polynomial in terms of the variable $\lambda=H_{1}$ leads to dispersionless Hirota equations, which express any second derivative $\frac{\partial^{2} \mathcal{F}}{\partial t_{k} \partial t_{n}}$

with arbitrary $k$ and $l$ in terms of the second derivatives $\frac{\partial^{2} \mathcal{F}}{\partial t_{k} \partial t_{1}}$ where one of the indices is fixed and corresponds to the first time. From formulas (4) one finds that

$$
d S \underset{\xi \rightarrow \infty}{=} \sum\left(k t_{k} \xi^{k-1} d \xi+\frac{\partial \mathcal{F}}{\partial t_{k}} \frac{d \xi}{\xi^{k+1}}\right)
$$

which is just an expansion in local co-ordinate at the marked point $P_{0}$. Taking the time derivatives (cf. with (8)) gives the set

$$
H_{k}=\frac{\partial S}{\partial t_{k}}=\xi^{k}-\sum_{j} \frac{\partial^{2} \mathcal{F}}{\partial t_{k} \partial t_{j}} \frac{1}{j \xi^{j}}=\xi^{k}(\lambda)_{+}, \quad k>0
$$

which forms a basis of meromorphic functions with poles at the point $P_{0}$, or just a particular polynomial basis, explicitly fixed by last equation. The set of the powers $\lambda^{k}$ has the same singularities as the set of functions (12), i.e. these two are related by simple linear transformation, e.g.

$$
\begin{gathered}
H_{1}=\lambda, \quad H_{2}=\lambda^{2}+2 \frac{\partial^{2} \mathcal{F}}{\partial t_{1}^{2}}, \\
H_{3}=\lambda^{3}+3 \frac{\partial^{2} \mathcal{F}}{\partial t_{1}^{2}} \lambda+\frac{3}{2} \frac{\partial^{2} \mathcal{F}}{\partial t_{1} \partial t_{2}}, \quad \ldots
\end{gathered}
$$

These equalities follow from the comparison of the singular at $P_{0}$ part of their expansions in $\xi$, following from (12). Comparing the negative "tails" of the expansion in $\xi$ of both sides of 
eq. (13) expresses the derivatives $\frac{\partial^{2} \mathcal{F}}{\partial t_{k} \partial t_{l}}$ (of $H_{k}$ in the l.h.s.) in terms of only those with $k=1$ (of $\lambda=H_{1}$ in the r.h.s.). These relations are called the dispersionless KP, or the dKP Hirota equations, e.g.

$$
\begin{aligned}
& \frac{\partial^{2} \mathcal{F}}{\partial t_{1} \partial t_{3}}=\frac{3}{8} X_{0}^{2}=\frac{3}{2}\left(\frac{\partial^{2} \mathcal{F}}{\partial t_{1}^{2}}\right)^{2} \\
& \frac{\partial^{2} \mathcal{F}}{\partial t_{3} \partial t_{3}}=\frac{3}{8} X_{0}^{3}=3\left(\frac{\partial^{2} \mathcal{F}}{\partial t_{1}^{2}}\right)^{3}
\end{aligned}
$$

We have listed here those, which will be of some interest for two-dimensional quantum gravity.

\subsection{Scaling}

Under the scaling $X \rightarrow \Lambda^{p} X, Y \rightarrow \Lambda^{q} Y$, (induced by $\lambda \rightarrow \Lambda \lambda$ and therefore $\xi \rightarrow \Lambda \xi$ ), the times (4) transform as $t_{k} \rightarrow \Lambda^{p+q-k} t_{k}$. Then from the second formula of (4) it follows that the function $\mathcal{F}$ scales as $\mathcal{F} \rightarrow \Lambda^{2(p+q)} \mathcal{F}$, or, for example, as

$$
\mathcal{F} \propto t_{1}^{2 \frac{p+q}{p+q-1}} f\left(\tau_{k}\right)
$$

where $f$ is supposed to be a scale-invariant function of corresponding dimensionless ratios of

the times $\tau_{k}=t_{k} / t_{1}^{\frac{p+q-m}{p+q-1}}(4)$. In the simplest $(p, q)=(2,2 K-1)$ case of dispersionless $\mathrm{KdV}$ one also expects a natural scaling of the form

$$
\mathcal{F} \propto\left(t_{2 K-3}\right)^{K+\frac{1}{2}} \mathbf{f}\left(\mathbf{t}_{l}\right)
$$

with $\mathrm{t}_{l}=t_{2 l-1} /\left(t_{2 K-3}\right)^{(K-l+1) / 2}$, where the role of cosmological constant is played by the time $t_{2 K-3} \propto \Lambda^{4}$.

\section{$2.4 \mathrm{KdV}$ series}

More explicit formulas can be written for the "KdV-series" $(p, q)=(2,2 K-1)$, corresponding to the $p=2 \mathrm{KdV}$ reduction of the KP hierarchy. Now

$$
\begin{gathered}
X=\lambda^{2}+2 u, \quad \xi=\sqrt{X}=\sqrt{\lambda^{2}+2 u} \\
Y=\lambda^{2 K-1}+\sum_{k=1}^{K-1} y_{k} \lambda^{2 k-1}
\end{gathered}
$$

and the explicit formula (10) reads

$$
S=\sum_{k=1}^{K+1} t_{2 k-1} X^{k-1 / 2}(\lambda)_{+}
$$


Dependence on $u$ upon the flat times is determined by a single equation

$$
\left.d S\right|_{d X=0}=0
$$

since $d X=2 \lambda d \lambda$ has the only zero at $\lambda=0$, or vanishing of the polynomial

$$
\left.P(u) \equiv \frac{1}{2} \frac{d S}{d \lambda}\right|_{\lambda=0}=\sum_{k=0}^{K} \frac{(2 k+1) ! !}{k !} t_{2 k+1} u^{k}=0
$$

Integrating square of the polynomial (20)

$$
\mathcal{F}=\frac{1}{2} \int_{0}^{u} P^{2}(v) d v=\frac{1}{2} \sum_{k, l=0}^{K} t_{2 k+1} t_{2 l+1} \frac{(2 k+1) ! !(2 l+1) ! !}{k ! l !(k+l+1)} u^{k+l+1}
$$

one gets the string free energy - the logarithm of quasiclassical tau-function, due to the formula

$$
\mathcal{F}=\frac{1}{2} \sum_{k, l} t_{k} t_{l} \operatorname{res}_{P_{0}}\left(\xi^{k} d H_{l}\right)
$$

expressing free energy [14] in terms of its second derivatives, and since the coefficient in the r.h.s. of (21) exactly coincides with the second derivative (6)

$$
\begin{gathered}
\operatorname{res}_{\lambda=\infty}\left(\xi^{2 k+1} d H_{2 l+1}\right)= \\
=\sum_{n \geq 0} \sum_{m=0}^{l} \frac{(2 u)^{n+m}}{n ! m !} \frac{\Gamma(k+3 / 2) \Gamma(l+3 / 2)(2(l-m)+1)}{\Gamma(k+3 / 2-n) \Gamma(l+3 / 2-m)} \operatorname{res}_{\lambda=\infty}\left(d \lambda \lambda^{2(k+l-n-m)+1}\right)= \\
=(2 u)^{k+l+1} \Gamma(k+3 / 2) \Gamma(l+3 / 2) \frac{2}{\pi} \sum_{m=0}^{l} \frac{(-)^{l-m}}{m !(k+l+1-m) !}= \\
=\frac{(2 k+1) ! !(2 l+1) ! !}{k ! l !(k+l+1)} u^{k+l+1}
\end{gathered}
$$

where the last equality holds, in particular, due to binomial identity $\sum_{m=0}^{l}(-)^{m}\left(\begin{array}{c}s \\ n\end{array}\right)=(-)^{l}\left(\begin{array}{c}s-1 \\ l\end{array}\right)$.

\section{Examples: particular (p,q) models}

\subsection{Pure gravity: the explicit partition function}

In this case $(p, q)=(2,3)$, one has only two nontrivial parameters $t_{1}$ and $t_{3}$, and the partition function can be calculated explicitly. The times (4) are expressed by

$$
\begin{gathered}
t_{5}=\frac{2}{5}, \quad t_{3}=\frac{2}{3} Y_{1}-X_{0}, \quad t_{1}=\frac{3}{4} X_{0}^{2}-X_{0} Y_{1} \\
t_{4}=\frac{1}{2} Y_{2}, \quad t_{2}=Y_{0}-Y_{2} X_{0}
\end{gathered}
$$


in terms of the coefficients of the polynomials

$$
X=\lambda^{2}+X_{0}, \quad Y=\lambda^{3}+Y_{2} \lambda^{2}+Y_{1} \lambda+Y_{0}
$$

The odd times $t_{1}, t_{3}$ and $t_{5}$ do not depend upon the even coefficients $Y_{0}$ and $Y_{2}$ of the second polynomial in (25), and in what follows we choose $Y_{2}=Y_{0}=0$, ensuring $t_{2}=t_{4}=0$. Relations (24) can be then easily solved for the latter coefficients of

$$
X=\lambda^{2}+X_{0}, \quad Y=\lambda^{3}+Y_{1} \lambda
$$

giving rise to

$$
X_{0}=\frac{1}{3} \sqrt{9 t_{3}^{2}-12 t_{1}}-t_{3}, \quad Y_{1}=\frac{1}{2} \sqrt{9 t_{3}^{2}-12 t_{1}}
$$

The second half of residues (4) gives

$$
\begin{gathered}
\frac{\partial \mathcal{F}}{\partial t_{1}}=\frac{1}{8} X_{0}^{3}-\frac{1}{4} Y_{1} X_{0}^{2} \\
\frac{\partial \mathcal{F}}{\partial t_{3}}=-\frac{1}{8} Y_{1} X_{0}^{3}+\frac{3}{64} X_{0}^{4}
\end{gathered}
$$

This results in the following explicit formula for the quasiclassical tau-function

$$
\mathcal{F}=\frac{1}{3240}\left(9 t_{3}^{2}-12 t_{1}\right)^{5 / 2}+\frac{1}{4} t_{3}^{3} t_{1}-\frac{1}{4} t_{3} t_{1}^{2}-\frac{3}{40} t_{3}^{5}
$$

At $t_{3} \rightarrow \infty$ (expansion at $t_{1} \rightarrow 0$ ) formula (29) gives

$$
\mathcal{F} \underset{t_{3} \rightarrow \infty}{=}-\frac{t_{1}^{3}}{18 t_{3}}\left(1+O\left(\frac{t_{1}}{t_{3}^{2}}\right)\right)
$$

which is the partition function of the Kontsevich model [17, 18] (also identified with the $(2,1)$ point or topological gravity). At $t_{1} \rightarrow \infty$ tau function (29) scales as $\mathcal{F} \propto t_{1}^{5 / 2}$ or partition function of pure two-dimensional gravity: expansion at $t_{1} \rightarrow \infty$ gives

$$
\mathcal{F}=\left(-3 t_{1}\right)^{5 / 2}\left(\frac{4}{405}-\frac{1}{54} \frac{t_{3}^{2}}{t_{1}}+\frac{1}{96} \frac{t_{3}^{4}}{t_{1}^{2}}+O\left(\frac{t_{3}^{6}}{t_{1}^{3}}\right)\right)+\ldots
$$

modulo analytic terms.

Formula (29) is the only example of exact computation. For the rest one needs to solve perturbatively the nonlinear string equation. It contains the polynomial part, which gives contribution only to a finite number of correlation functions. Usually such "non-universal" part is neglected, when comparing the result of the computation with the world-sheet Liouville theory. It also vanishes at $t_{3}=0$ or at vanishing of the time, corresponding to so called "boundary operator" (see e.g. [21]), the $t_{2 K-1}$ variable in the $(2,2 K-1) \mathrm{KdV}$ series, which we shall usually neglect in what follows. However, these terms are essential, when taking the limit (30) to the topological Kontevich model, and it means that they come from the contact terms of topological origin. 


\subsection{The gravitational Yang-Lee model: $(\mathrm{p}, \mathrm{q})=(2,5)$}

The calculation of times according to (4) gives

$$
\begin{gathered}
t_{1}=-\frac{5}{8} X_{0}^{3}+\frac{3}{4} Y_{3} X_{0}^{2}-Y_{1} X_{0} \\
t_{3}=\frac{5}{4} X_{0}^{2}-Y_{3} X_{0}+\frac{2}{3} Y_{1} \\
t_{5}=\frac{2}{5} Y_{3}-X_{0} \\
t_{7}=\frac{2}{7}
\end{gathered}
$$

for the polynomials

$$
\begin{gathered}
X=\lambda^{2}+X_{0} \\
Y=\lambda^{5}+Y_{3} \lambda^{3}+Y_{1} \lambda
\end{gathered}
$$

These equations are easily solved for

$$
\begin{gathered}
Y_{1}=\frac{3}{2}\left(t_{3}+\frac{5}{4} X_{0}^{2}+\frac{5}{2} t_{5} X_{0}\right) \\
Y_{3}=\frac{5}{2}\left(X_{0}+t_{5}\right)
\end{gathered}
$$

ending up with the only nonlinear string equation for $X_{0}$

$$
t_{1}=-\frac{5}{8} X_{0}^{3}-\frac{3}{2} t_{3} X_{0}
$$

The one-point functions (4) are given by

$$
\begin{aligned}
\frac{\partial \mathcal{F}}{\partial t_{1}} & =-\frac{15}{64} X_{0}^{4}-\frac{3}{8} t_{3} X_{0}^{2} \\
\frac{\partial \mathcal{F}}{\partial t_{3}} & =-\frac{9}{64} X_{0}^{5}-\frac{3}{16} t_{3} X_{0}^{3}
\end{aligned}
$$

while the two-point functions are

$$
\frac{\partial^{2} \mathcal{F}}{\partial t_{1}^{2}}=\frac{X_{0}}{2}, \quad \frac{\partial^{2} \mathcal{F}}{\partial t_{1} \partial t_{3}}=\frac{3}{8} X_{0}^{2}, \quad \frac{\partial^{2} \mathcal{F}}{\partial t_{3} \partial t_{3}}=\frac{3}{8} X_{0}^{3}
$$

The last expressions can be obtained by differentiation (36) upon following from (35) explicit formulas for $\frac{\partial X_{0}}{\partial t_{1}}$ and $\frac{\partial X_{0}}{\partial t_{3}}$, or they follow directly from the Hirota equations (14).

To compare the predictions of the "integrable" approach for correlators in two-dimensional gravity with the calculations in world-sheet theory, one needs first to make certain correspondences in the space of coupling constants. The simplest one comes from the scaling properties (15), (16). In the Yang-Lee theory the role of the cosmological constant is played 
by the KdV time $t_{3}$, and from the scaling properties of the "fixed area" partition function $F_{A}\left(t_{1}\right)=A^{-7 / 2} \mathbf{z}\left(t_{1} A^{3 / 2}\right)$ (cf. with [19]) one gets for the Laplace transformed $\mathcal{F}\left(t_{1}, t_{3}\right)=$ $\int_{0}^{\infty} \frac{d A}{A} e^{-t_{3} A} F_{A}\left(t_{1}\right)$, or

$$
\begin{gathered}
\mathcal{F}=t_{3}^{7 / 2} \mathrm{f}\left(\frac{t_{1}}{t_{3}^{3 / 2}}\right) \equiv t_{3}^{7 / 2} \mathrm{f}(\mathrm{t}) \\
\frac{\partial \mathcal{F}}{\partial t_{1}}=t_{3}^{2} \mathrm{f}^{\prime}, \quad \frac{\partial^{2} \mathcal{F}}{\partial t_{1}^{2}}=t_{3}^{1 / 2} \mathrm{f}^{\prime \prime}, \quad \ldots
\end{gathered}
$$

and the string equation turns into

$$
\mathrm{t}+5\left(\mathrm{f}^{\prime \prime}\right)^{3}+3 \mathrm{f}^{\prime \prime}=0
$$

to be solved for the coefficients $\left.\mathrm{f}_{n} \equiv \mathrm{f}^{(n)}\right|_{\mathrm{t}=0}$ in the expansion of

$$
\mathcal{F}=t_{3}^{7 / 2} \mathrm{f}_{0}+t_{1} t_{3}^{2} \mathrm{f}_{1}+\frac{t_{1}^{2} t_{3}^{1 / 2}}{2} \mathrm{f}_{2}+\frac{t_{1}^{3}}{6 t_{3}} \mathrm{f}_{3}+\ldots
$$

which gives rise to rational expressions

$$
\begin{gathered}
\mathrm{f}_{3}=-\frac{1}{3\left(1+5 \mathrm{f}_{2}^{2}\right)}, \quad \mathrm{f}_{4}=-\frac{10 \mathrm{f}_{2}}{9\left(1+5 \mathrm{f}_{2}^{2}\right)^{3}}, \quad \mathrm{f}_{5}=\frac{10\left(1-25 \mathrm{f}_{2}^{2}\right)}{27\left(1+5 \mathrm{f}_{2}^{2}\right)^{5}} \\
\mathrm{f}_{6}=\frac{1000 \mathrm{f}_{2}\left(1-10 \mathrm{f}_{2}^{2}\right)}{81\left(1+5 \mathrm{f}_{2}^{2}\right)^{7}}, \quad \mathrm{f}_{7}=-\frac{1000\left(1-95 \mathrm{f}_{2}^{2}+550 \mathrm{f}_{2}^{4}\right)}{243\left(1+5 \mathrm{f}_{2}^{2}\right)^{9}} \\
\mathrm{f}_{8}=-\frac{70000 \mathrm{f}_{2}\left(2-70 \mathrm{f}_{2}^{2}+275 \mathrm{f}_{2}^{4}\right)}{2187\left(1+5 \mathrm{f}_{2}^{2}\right)^{11}}, \quad \ldots
\end{gathered}
$$

in terms of the two-point function $\mathrm{f}_{2}$, which itself can be found as a nonvanishing solution to the "reduced" string equation

$$
3 \mathrm{f}_{2}+5 \mathrm{f}_{2}^{3}=0
$$

The "total normalization" $\mathrm{f}_{0}$ and the "one-point function" $\mathrm{f}_{1}$, which does not have a universal sense, since it is coupled to an analytic term in the expansion (40), in principle are determined by residue formula for $\partial \mathcal{F} / \partial t_{3}$, or

$$
7 f-3 t f^{\prime}+9\left(f^{\prime \prime}\right)^{5}+3\left(f^{\prime \prime}\right)^{3}=0
$$

giving rise to

$$
\begin{gathered}
\mathrm{f}_{0}=-\frac{9}{7} \mathrm{f}_{2}^{5}-\frac{3}{7} \mathrm{f}_{2}^{3}=-\frac{3}{7} \mathrm{f}_{2}^{3}\left(1+3 \mathrm{f}_{2}^{2}\right) \\
\mathrm{f}_{1}=-\frac{9}{4} \mathrm{f}_{2}^{3} \mathrm{f}_{3}-\frac{45}{4} \mathrm{f}_{2}^{4} \mathrm{f}_{3}=\frac{3}{4} \mathrm{f}_{2}^{2}
\end{gathered}
$$

This results in the rational "invariant ratios", e.g.

$$
\begin{gathered}
\frac{\mathrm{f}_{4} \mathrm{f}_{2}}{\mathrm{f}_{3}^{2}}=-3, \quad \frac{\mathrm{f}_{4} \mathrm{f}_{3}}{\mathrm{f}_{2} \mathrm{f}_{5}}=-\frac{1}{8}, \quad \frac{\mathrm{f}_{2} \mathrm{f}_{4}}{\mathrm{f}_{0} \mathrm{f}_{6}}=1, \quad \frac{\mathrm{f}_{4}^{2}}{\mathrm{f}_{0} \mathrm{f}_{8}}=-\frac{6}{143}, \\
\frac{\mathrm{f}_{2}^{2}}{\mathrm{f}_{0} \mathrm{f}_{4}}=\frac{\mathrm{f}_{2} \mathrm{f}_{6}}{\mathrm{f}_{4}^{2}}=-35
\end{gathered}
$$


to be possibly compared with the computations in the world-sheet theory.

\subsection{Mixing in $(2,7)$ model}

The $(p, q)=(2,7)$ model naively is not much different from the Yang-Lee case of $(2,5)$ theory considered in sect. 3.2. The polynomials (1) are

$$
\begin{gathered}
X=\lambda^{2}+X_{0} \\
Y=\lambda^{7}+\frac{7 X_{0}}{2} \lambda^{5}+Y_{3} \lambda^{3}+Y_{1} \lambda
\end{gathered}
$$

and the calculation of flat times (4) gives

$$
\begin{gathered}
t_{1}=\frac{3}{4} Y_{3} X_{0}^{2}-\frac{105}{64} X_{0}^{4}-Y_{1} X_{0} \\
t_{3}=\frac{35}{12} X_{0}^{3}-Y_{3} X_{0}+\frac{2}{3} Y_{1} \\
t_{5}=-\frac{7}{4} X_{0}^{2}+\frac{2}{5} Y_{3} \\
t_{7}=0 \\
t_{9}=\frac{2}{9}
\end{gathered}
$$

Again we see, that (47) can be easily solved w.r.t. $Y_{j}$, but the only coefficient $X_{0}$ now satisfies

$$
t_{1}=-\frac{35}{64} X_{0}^{4}-\frac{15}{8} t_{5} X_{0}^{2}-\frac{3}{2} t_{3} X_{0}
$$

where we put $t_{7}=0$ for the coefficient at the "boundary" operator [21].

The one-point functions (4) are given for the $(2,7)$ model by

$$
\begin{gathered}
\frac{\partial \mathcal{F}}{\partial t_{1}}=-\frac{7}{32} X_{0}^{5}-\frac{1}{4} Y_{1} X_{0}^{2}+\frac{1}{8} Y_{3} X_{0}^{3}=-\frac{7}{32} X_{0}^{5}-\frac{5}{8} X_{0}^{3} t_{5}-\frac{3}{8} X_{0}^{2} t_{3} \\
\frac{\partial \mathcal{F}}{\partial t_{3}}=-\frac{1}{8} Y_{1} X_{0}^{3}-\frac{35}{512} X_{0}^{6}+\frac{3}{64} Y_{3} X_{0}^{4}=-\frac{35}{256} X_{0}^{6}-\frac{45}{128} X_{0}^{4} t_{5}-\frac{3}{16} X_{0}^{3} t_{3} \\
\frac{\partial \mathcal{F}}{\partial t_{5}}=-\frac{15}{512} X_{0}^{7}-\frac{5}{64} Y_{1} X_{0}^{4}+\frac{3}{128} Y_{3} X_{0}^{5}=-\frac{25}{256} X_{0}^{7}-\frac{15}{64} X_{0}^{5} t_{5}-\frac{15}{128} X_{0}^{4} t_{3}
\end{gathered}
$$

In the r.h.s.'s of (49) we already substituted the expressions for $Y_{j}$ in terms of times (47), and the rest is to solve (48) by expanding in $t_{3}$ and $t_{5}$ and substitute result into (49).

The scaling anzatz (16), (38) now reads

$$
\begin{gathered}
\mathcal{F}=t_{5}^{9 / 2} \mathrm{f}\left(\frac{t_{1}}{t_{5}^{2}}, \frac{t_{3}}{t_{5}^{3 / 2}}\right) \\
\frac{\partial \mathcal{F}}{\partial t_{1}}=t_{5}^{5 / 2} \mathrm{f}^{(1)}, \quad \frac{\partial^{2} \mathcal{F}}{\partial t_{1}^{2}}=\frac{X_{0}}{2}=t_{5}^{1 / 2} \mathrm{f}^{(11)}, \ldots
\end{gathered}
$$


where we have introduced shorten notation for the derivatives over the first argument of $f\left(t_{1}, t_{2}\right)$, and string equation (48) turns into

$$
\mathrm{t}_{1}+\frac{35}{4} \mathrm{u}^{4}+\frac{15}{2} \mathrm{u}^{2}+3 \mathrm{t}_{2} \mathrm{u}=0
$$

for $u=f^{(11)}$.

Expansion should be considered in the vicinity of the point $t_{1}=\frac{25}{28} t_{5}^{2}$, where the one-point function in the first equation of (49) vanishes on string equation (48) at $t_{3}=0$. It means, in particular, that the function $f$ should be expanded around the non-vanishing background value $\mathrm{t}_{1}=\frac{25}{28}$ of its first argument.

\subsection{Ising model $(\mathrm{p}, \mathrm{q})=(3,4)$}

The residue formulas for the polynomials

$$
\begin{gathered}
X=\lambda^{3}+X_{1} \lambda+X_{0} \\
Y=\lambda^{4}+Y_{2} \lambda^{2}+Y_{1} \lambda+Y_{0}
\end{gathered}
$$

give rise to

$$
\begin{gathered}
Y_{2}=\frac{4}{3} X_{1}+\frac{5}{3} t_{5} \\
Y_{0}=\frac{2}{9} X_{1}^{2}+\frac{10}{9} X_{1} t_{5} \\
Y_{1}=\frac{4}{3} X_{0}
\end{gathered}
$$

(where the last equation is true upon $t_{4}=0$ ), while $X_{0}$ and $X_{1}$ satisfy

$$
\begin{gathered}
t_{1}=-\frac{2}{3} X_{0}^{2}+\frac{4}{27} X_{1}^{3}+\frac{5}{9} t_{5} X_{1}^{2} \\
t_{2}=-\frac{2}{3} X_{0} X_{1}-\frac{5}{3} t_{5} X_{0}
\end{gathered}
$$

Differentiating equations (54) one can find explicitly expressions for the first derivatives

$$
\frac{\partial X_{1}}{\partial t_{j}}=\frac{Q_{1}^{(j)}}{R}, \quad \frac{\partial X_{0}}{\partial t_{j}}=\frac{Q_{0}^{(j)}}{R}, \quad j=1,2,5
$$

with $R=4 X_{1}^{3}+12 X_{0}^{2}+20 t_{5} X_{1}^{2}+25 t_{5}^{2} X_{1}$ and

$$
\begin{gathered}
Q_{1}^{(1)}=\frac{9}{2}\left(2 X_{1}+5 t_{5}\right), \quad Q_{0}^{(1)}=-9 X_{0} \\
Q_{1}^{(2)}=-18 X_{0}, \quad Q_{0}^{(2)}=-3 X_{1}\left(2 X_{1}+5 t_{5}\right) \\
Q_{1}^{(5)}=-\frac{5}{2}\left(2 X_{1}^{3}+5 t_{5} X_{1}^{2}+12 X_{0}^{2}\right), \quad Q_{0}^{(5)}=-5 X_{1} X_{0}\left(X_{1}+5 t_{5}\right)
\end{gathered}
$$


Solving the second equation of (54) for $X_{0}$ and substituting the result into the first one, turns it into the Boulatov-Kazakov equation for $X_{1}[20]$

$$
t_{1}=-\frac{6 t_{2}^{2}}{\left(2 X_{1}+5 t_{5}\right)^{2}}+\frac{4}{27} X_{1}^{3}+\frac{5}{9} t_{5} X_{1}^{2}
$$

(contains information about all singularities of $\mathcal{F}$ for arbitrary magnetic field $t_{2}$ and fermion mass $\left.t_{5}\right)$.

It is interesting to compare the Boulatov-Kazakov equation with what gives here formula (19). The branch points are given by $d X=0$ for the first polynomial from (52), or $\lambda_{ \pm}=$ $\pm \sqrt{-\frac{X_{1}}{3}}$, so that vanishing of the derivative of function

$$
S=t_{1} X(\lambda)_{+}^{1 / 3}+t_{2} X(\lambda)_{+}^{2 / 3}+t_{5} X(\lambda)_{+}^{5 / 3}+\frac{3}{7} X(\lambda)_{+}^{7 / 3}
$$

at $\lambda_{ \pm}$or $\left.S^{\prime}(\lambda)\right|_{\lambda_{+}}=\left.S^{\prime}(\lambda)\right|_{\lambda_{-}}$gives rise to the last equation of (54), to be easily solved for $X_{0}$. Substituting the result into $\left.S^{\prime}(\lambda)\right|_{\lambda_{+}}+\left.S^{\prime}(\lambda)\right|_{\lambda_{-}}=0$ reproduces immediately the string equation (57).

The one-point functions

$$
\begin{gathered}
\frac{\partial \mathcal{F}}{\partial t_{1}}=\frac{1}{27} X_{1}^{4}+\frac{10}{81} t_{5} X_{1}^{3}-\frac{4}{9} X_{1} X_{0}^{2}-\frac{5}{9} t_{5} X_{0}^{2} \\
\frac{\partial \mathcal{F}}{\partial t_{2}}=\frac{4}{27} X_{1}^{3} X_{0}+\frac{10}{27} t_{5} X_{1}^{2} X_{0}-\frac{8}{27} X_{0}^{3} \\
\frac{\partial \mathcal{F}}{\partial t_{5}}=\frac{40}{243} X_{1}^{3} X_{0}^{2}-\frac{10}{2187} X_{1}^{6}+\frac{25}{81} t_{5} X_{1}^{2} X_{0}^{2}-\frac{10}{729} t_{5} X_{1}^{5}-\frac{5}{27} X_{0}^{4}
\end{gathered}
$$

give rise to

$$
\begin{gathered}
\frac{\partial^{2} \mathcal{F}}{\partial t_{1}^{2}}=\frac{X_{1}}{3}, \quad \frac{\partial^{2} \mathcal{F}}{\partial t_{1} \partial t_{2}}=\frac{2 X_{0}}{3} \\
\frac{\partial^{2} \mathcal{F}}{\partial t_{1} \partial t_{5}}=\frac{5}{9} X_{0}^{2}-\frac{5}{81} X_{1}^{3}, \quad \frac{\partial^{2} \mathcal{F}}{\partial t_{2} \partial t_{5}}=-\frac{10}{27} X_{1}^{2} X_{0}, \\
\frac{\partial^{2} \mathcal{F}}{\partial t_{2}^{2}}=-\frac{2}{9} X_{1}^{2}, \quad \frac{\partial^{2} \mathcal{F}}{\partial t_{5}^{2}}=-\frac{50}{81} X_{1}^{2} X_{0}^{2}+\frac{5}{243} X_{1}^{5}, \quad \ldots
\end{gathered}
$$

At $t_{2}=0$ one gets for the one-point functions (61)

$$
\begin{gathered}
\left.\frac{\partial \mathcal{F}}{\partial t_{1}}\right|_{t_{2}=0}=\frac{1}{27} X_{1}^{4}+\frac{10}{81} t_{5} X_{1}^{3},\left.\quad \frac{\partial \mathcal{F}}{\partial t_{2}}\right|_{t_{2}=0}=0 \\
\left.\frac{\partial \mathcal{F}}{\partial t_{5}}\right|_{t_{2}=0}=-\frac{10}{729} X_{1}^{5}\left(t_{5}+\frac{X_{1}}{3}\right)
\end{gathered}
$$


Note also, that for $t_{2}=0$ the second equation of (54) has the only reasonable solution $X_{0}=0$, while the first one turns into

$$
t_{1}=\frac{4}{27} X_{1}^{3}+\frac{5}{9} t_{5} X_{1}^{2}
$$

which almost coincides with the perturbation of the Yang-Lee $(2,5)$ model by quadratic term (cf. with (35) and note that potential $\frac{X_{1}}{3}$ from (62) is an analog of $\frac{X_{0}}{2}$ from (35), see (37) and (60)). More strictly, the quadratic term can be removed by redefinition

$$
\begin{gathered}
\hat{t}_{1}=\frac{4}{27} \hat{X}_{1}^{3}-\frac{25}{36} t_{5}^{2} \hat{X}_{1} \\
\hat{t}_{1}=t_{1}-\frac{125}{216} t_{5}^{3}, \quad \hat{X}_{1}=X_{1}+\frac{5}{4} t_{5}
\end{gathered}
$$

This redefinition exactly fits [21] the vanishing of the energy 3-point function in the Ising model. Indeed, if one identifies $\hat{t}_{1}$ with the cosmological constant of the world-sheet theory, the energy 3-point functions

$$
\left.\frac{\partial^{3}}{\partial t_{5}^{3}} \mathcal{F}\left(\hat{t}_{1}+C t_{5}^{3}, t_{2}=0, t_{5}\right)\right|_{t_{5}=0}=\left.\left(6 C \frac{\partial \mathcal{F}}{\partial t_{1}}+\frac{\partial^{3} \mathcal{F}}{\partial t_{5}^{3}}\right)\right|_{t_{2,5}=0}
$$

vanishes exactly at $C=\frac{125}{216}$. To calculate the r.h.s. of (64) one can use the first equation from (61) and differentiate the last formula from (60) using (55), which is quite easy since $X_{0}=0$ at $t_{2}=0$. An alternative and more fundamental way is to use directly the residue formula $(7)$ for the third derivatives, which gives here

$$
\frac{\partial^{3} \mathcal{F}}{\partial t_{5}^{3}}=\operatorname{res}_{d X=0}\left(\frac{d H_{5}^{3}}{d X d Y}\right)=\sum_{\lambda=\lambda_{ \pm}} \frac{H_{5}^{\prime}(\lambda)^{3}}{6 \lambda Y^{\prime}(\lambda)} \underset{t_{2}=t_{5}=0}{=}-\frac{125}{972} X_{1}^{4}
$$

It is interesting to point out, that under reparameterization (63) in the space of couplings

$$
\begin{aligned}
& X_{1}=\hat{X}_{1}-\frac{5}{4} t_{5} \\
& t_{1}=\hat{t}_{1}+\frac{125}{216} t_{5}^{3}
\end{aligned}
$$

the reduced string equation (62) acquires the form of (analytically continued) string equation (35) for the Yang-Lee model, with $t_{3}^{\mathrm{YL}} \sim t_{5}^{2}$ of the Yang-Lee model being substituted by the square of the $t_{5}=t_{5}^{\text {Ising }}$ of the (reduced) Ising model. However, one should use the scaling anzatz (15) rather than (16), has been used for the $(2,5)$ theory in (38), for the function $F\left(\hat{t}_{1}, t_{5}\right)=\left.\mathcal{F}\left(\hat{t}_{1}+\frac{125}{216} t_{5}^{3}, t_{5}\right)\right|_{t_{2}=0}$. Since

$$
\frac{\partial^{2} F}{\partial \hat{t}_{1}^{2}}=\hat{X}_{1}\left(\hat{t}_{1}, t_{5}^{2}\right)-\frac{5}{4} t_{5}
$$

as follows from string equation (63) for $\hat{X}_{1}$ and the couplings are dimensional, the gravitational Ising free energy $F=\hat{F}\left(\hat{t}_{1}, t_{5}^{2}\right)-\frac{5}{8} t_{5} \hat{t}_{1}^{2}$ is an even function of $t_{5}$, apart of an analytic cubic term, and its expansion gives all the $\left\langle\epsilon^{2 n}\right\rangle$ correlators of the gravitationally dressed $(3,4)$ Ising model. We shall comment more about the relation of these two models in the next section. 


\section{Ising versus Yang-Lee}

Both gravitational Ising model and $(2,5)$ Yang-Lee minimal theory arise as two different critical points in a system of Ising spins on a random lattice. Moreover, since both theories have $p+q=7$, they have identical scaling in the first KP variable (15), which originally has cause a confusion, when distinguishing these two minimal string theories. In particular, that origins from the fact that string equations of those two models can be obtained from each other by simple reparameterization in the space of couplings, as we have noticed already in the previous section.

However, the physical sense of parameters, arising in these two equations is totally different. One can say, that the same KP time variable has different "quantum numbers", when one takes a solution, corresponding to a different critical point. For example, the role of cosmological constant, coupled to a unity operator on world-sheet is played by $t_{1}$ in $(3,4)$ Ising, but by $t_{3}$ in the $(2,5)$ Yang-Lee theory. Below we shall try to present more details about this relation and describe it as much as possible from the point of view of (dispersionless) KP theory.

\subsection{Kostov equation}

This is a name, given by Alesha Zamolodchikov to a "phenomenological" transcendental equation, satisfied by the second derivative of free energy over the cosmological constant $u \sim \frac{\partial^{2} \mathcal{F}}{\partial x^{2}}$ of the form

$$
u^{\nu}+t u^{\nu-1}=x
$$

where $\nu=\nu(p, q)=\frac{p}{q-p}$. For the cases of interest one gets integer $\nu(2,3)=2$ for pure gravity and $\nu(3,4)=3$ for Ising (both are unitary with $q=p+1$ ), but $\nu(2,5)=\frac{2}{3}$.

Hence, for the Ising model the Kostov equation reads

$$
u_{I}^{3}+t_{I} u_{I}^{2}=x_{I}
$$

and coincides (after renormalization $x_{I} \sim t_{1}, t_{I} \sim t_{5}$ and $u_{I} \sim X_{1}=3 \frac{\partial^{2} \mathcal{F}}{\partial t_{1}^{2}}$, below in this section we shall use different normalization from conventional in KP theory, to get rid of ugly numerical constants) with the Boulatov-Kazakov equation (62) when $t_{2}=0$, i.e. for vanishing magnetic field.

For the Yang-Lee model equation (68) $u_{Y L}^{2 / 3}+t_{Y L} u_{Y L}^{-1 / 3}=x_{Y L}$ after the substitution $u_{Y L}=$ $v_{Y L}^{3}$ turns into

$$
v_{Y L}^{3}-x_{Y L} v_{Y L}=-t_{Y L}
$$

which coincides (again, up to a similar renormalization of couplings) with the Yang-Lee string equation (35) upon $t_{3} \sim x_{Y L}, t_{1} \sim t_{Y L}$ and $X_{0}=2 \frac{\partial^{2} \mathcal{F}}{\partial t_{1}^{2}} \sim v_{Y L}$. 
Comparing (70) with (69) one finds that, as we have already done in the previous section, one may indeed identify $u_{I}$ with $v_{Y L}$ after appropriate shifts of the variables (66) and pointing out the change of the quantum numbers: $t_{1} \sim x_{I} \sim t_{Y L}$ and $t_{3} \sim t_{I}^{2} \sim x_{Y L}$.

The relation $u_{Y L} \sim v_{Y L}^{3}$ is quite clear from the point of view of equations (14). It is just a particular Hirota equation for dispersionless KP hierarchy, expressing

$$
u_{Y L} \sim \frac{\partial^{2} \mathcal{F}}{\partial t_{3}^{2}}=3\left(\frac{\partial^{2} \mathcal{F}}{\partial t_{1}^{2}}\right)^{3} \sim v_{Y L}^{3}
$$

the function satisfying equation (68), and being here a double derivative of free energy w.r.t the third time of the hierarchy, in terms of the canonical KP potential, being always a double derivative w.r.t. the first time.

From the point of view of KP theory it is also rather clear, why equation (68) is applicable only for $p<q<2 p$, in particular only for $K=2,3$ with $\nu(2,2 K-1)=\frac{2}{2 K-3}$. When transforming it to conventional KdV string equation (20), like it was done in (70) for the YangLee model, one finds that the variable $t$ should be generally identified with $t_{7-2 K^{-}}$th time of KP hierarchy, which does not have clear sense at $K>3$.

\subsection{Zamolodchikov curve for Ising}

If all parameters of the gravitational Ising model are "alive", the best way is to study, following [19], the fixed area partition function

$$
\begin{gathered}
Z_{a}=\int \frac{d x}{2 \pi i a} u e^{x a}=-\int \frac{d u}{2 \pi i a^{2}} e^{x a} \\
x=u^{3}+\frac{3}{2} T u^{2}+\frac{H^{2}}{(u+T)^{2}}
\end{gathered}
$$

where we use again the rescaled variables $x=x_{I} \sim t_{1}, H \sim t_{2}, T \sim t_{5}$ and the rescaled Boulatov-Kazakov equation (57) for $u \sim \frac{\partial^{2} \mathcal{F}}{\partial x^{2}}$. The saddle point equation $\frac{d x}{d u}=0$ for the integral in (72) is given by

$$
u(u+T)^{4}=\frac{2}{3} H^{2} \equiv \xi^{2} T^{5}
$$

In rescaled variables $u \sim T U$, the saddle point equation (73) presents a Riemann surface

$$
U(U+1)^{4}=\xi^{2}
$$

which is a double-cover of the $U_{c}$-plane and a 5 -sheet cover of the "magnetic" plane $\xi$, and the function $x$ on the curve (74) contains description of all singularities in the gravitational Ising model [23]. 
In particular, the Yang-Lee singularity arises at critical value of magnetic field $H=H_{c}$, where two values $\xi_{c}=\sqrt{\frac{2}{3}} \frac{H_{c}}{T^{5 / 2}}= \pm i \frac{16}{5^{5 / 2}}$ correspond to two points on the curve $(74)$

$$
U_{c}=-\frac{1}{5}, \quad \xi_{c}^{2}=U_{c}\left(U_{c}+1\right)^{4}=-\frac{4^{4}}{5^{5}}
$$

where $\left.\frac{d \xi}{d U}\right|_{U=U_{c}}=0$. At the Yang-Lee point one also obviously has $\left.\frac{d^{2} x}{d U^{2}}\right|_{U=U_{c}}=0$, and

$$
x=-\frac{7}{50} T^{3}+\frac{5}{2}\left(U-U_{c}\right)^{3}+\ldots=x_{c}+\frac{625}{128} T^{3}\left(\frac{\xi^{2}-\xi_{c}^{2}}{2}\right)^{3 / 2}+\ldots
$$

so that $X \sim \frac{x-x_{c}}{T^{3}} \sim \mu^{3 / 2}$ scales as the right fractional power of the cosmological constant

$x_{Y L}=\mu \sim \xi-\xi_{c}$ in the Yang-Lee model, corresponding to the well-known scaling $t_{1} \sim t_{3}^{3 / 2}$ of $\mathrm{KP}$ times at the critical point with $p+q=7$. For the expansion of Boulatov-Kazakov equation one can now write

$$
\frac{X}{\epsilon^{3}} \sim \mu V+V^{3}+O(\epsilon)
$$

where $H-H_{c} \sim \epsilon^{2} \mu, U+\frac{1}{5} \sim \epsilon V$, i.e. the Zamolodchikov curve in the vicinity of the Yang-Lee singularity is described, up to renormalization of parameters, by string equation (35).

\section{Discussion}

We have tried to demonstrate in this paper, that all spherical correlation functions in quantum Liouville gravity are all contained and can be easily extracted from the "science of polynomials" - dispersionless KP hierarchy. A simple collection of residue formulas allows to extract the invariant ratios, to be further compared with the correlation functions in world-sheet theory, which can be now also computed - though in a much more cumbersome way - mostly due to the results of Alesha Zamolodchikov in Liouville theory.

Such application of classical integrable science to the problems of two-dimensional quantum physics is already a step towards dynamical physics from topological strings, where similar science has been already used with visible success, see e.g. [25, 17, 18, 26, 27, 28].

The most nontrivial point in application of this "integrable science" is its interpretation in terms of the world-sheet theory. The first point concerns resonances [21, 22], which allow nonlinear relations between the couplings in KP and world-sheet theories when the fractions of the KPZ scaling dimensions of couplings [2] are integer. We have observed this phenomenon in the cases of $(2,7)$ and $(3,4)$ minimal theories, where it is yet quite easy taken into account by just playing with the residue formulas for one- two- and three-point functions. Naively, these resonance reparameterizations look as particular $W$-flows in the space of couplings, but this question deserves further investigation. 
Another nontrivial point is related to the fact that KP times may have different physical sense, or different quantum numbers, when we expand a KP solution around different backgrounds, corresponding to particular minimal theories. We have noticed this, say, when comparing the formulations for the gravitational Ising and Yang-Lee models. This is the simplest observation for a very important generic fact that physical observables may change their quantum numbers, when effective field theory is moved in the moduli space - like in four-dimensional supersymmetric gauge theories electrically charged objects may capture magnetic charges and vice versa. Two dimensional quantum gravity is therefore a good laboratory for studying such effects.

Finally, let us say few words, how the picture of dispersionless KP for the minimal string theory could be deformed towards quasiclassical hierarchies of generic nonsingular type. An invariant way to look at the basic polynomials $X=\lambda^{p}+\ldots$ and $Y=\lambda^{q}+\ldots$ (1) is to say, that they satisfy an algebraic equation

$$
Y^{p}-X^{q}-\sum f_{i j} X^{i} Y^{j}=0
$$

with some particular coefficients $\left\{f_{i j}\right\}$. Generally, for arbitrary coefficients this is a smooth curve of genus

$$
g=\frac{(p-1)(q-1)}{2}
$$

which is a resolution or desingularization of the situation, when $X$ and $Y$ can be parameterized as polynomial of a uniformizing global variable $\lambda$. This number coincides with the number of primaries in corresponding minimal conformal $(p, q)$ theory. Such curves can be obtained, say, by reduction of the curve of two-matrix model [24]. An interesting example is the hyperelliptic curve of the $(2,7)$ model $Y^{2}=X^{7}+\sum_{j=0}^{5} f_{j} X^{j}$, satisfied by (46) with the coefficients $f_{j}=f_{j}\left(X_{0}, Y_{1}, Y_{3}\right), j=0, \ldots, 5$. At vanishing times (except for $t_{9}=2 / 9$, see (47)), it shrinks to a cusp $Y^{2}=X^{7}$, or $Y^{2}=X^{3}\left(X^{2}+\frac{5 t_{5}}{2}\right)^{2}$ for nonvanishing cosmological $t_{5}$, which is however "resolved" by passing to worldsheet times $t_{1} \rightarrow t_{1}+\frac{25}{28} t_{5}^{2}$ as $Y^{2}=(X-u)\left(X^{3}+\frac{u}{2} X^{2}-\frac{u^{2}}{2} X-\frac{u^{3}}{8}\right)^{2}$ with $u^{2}=-\frac{20 t_{5}}{7}$. This form directly generalizes the curve of the Yang-Lee model $Y^{2}=(X-u)\left(X^{2}-\frac{u}{2} X+\frac{u^{2}}{4}\right)^{2}$ for (33) with $u^{2}=-\frac{12 t_{3}}{5}$ proportional to nonvanishing cosmological time.

For such curves, the residue formulas we have discussed above should be extended by period integrals $\oint Y d X$ along all nontrivial cycles on the curve (78). The sense of such period integrals is analogous to the Seiberg-Witten periods or the filling fractions in matrix models. As usually in quasiclassical hierarchies, the appearance of corresponding period variables reflect increasing number of unfrozen coefficients in the equation (78) or new deformations of the background of the minimal string theories. The study of such deformations is again a long-standing, but still an open problem.

Acknowledgements. Most of my understanding of the delicate issues of the relation among different two-dimensional quantum gravity models has come after conversations with Alesha 
Zamolodchikov, possessing very deep feeling of the subject and amazing intuition. I am also grateful to A. Belavin, S. Kharchev, I. Krichever, A. Zabrodin and A. Zamolodchikov for useful discussions. The work was partially supported by the Federal Nuclear Energy Agency, the RFBR grant 08-01-00667, the grant for support of Scientific Schools LSS-1615.2008.2, the INTAS grant 05-1000008-7865, the project ANR-05-BLAN-0029-01, the NWO-RFBR program 047.017.2004.015, the Russian-Italian RFBR program 06-01-92059-CE, and by the Dynasty foundation. I am also grateful for warm hospitality to SUBATECH in Nantes and IHES in Bur-sur-Yvette, where this work has been completed.

\section{References}

[1] A.Polyakov, Phys.Lett. B103(1981) 207-210; Phys.Lett. B103(1981) 211-214.

[2] A.Polyakov, Mod.Phys.Lett. A2 (1987) 893-898;

V.Knizhnik, A.Polyakov and A.Zamolodchikov, Mod.Phys.Lett. A3 (1988) 819-826.

[3] F.David, Mod.Phys.Lett. A3 (1988) 1651-1659;

J.Distler and H.Kawai, Nucl.Phys. B231 (1989) 509-528.

[4] J.Ambjørn, B.Durhuus and J.Frölich, Nucl.Phys. B257[FS14] (1985) 433;

F.David, Nucl.Phys. B257[FS14] (1985) 45;

V.Kazakov, Phys.Lett. 150B (1985) 282;

V.Kazakov, I.Kostov and A.Migdal, Phys.Lett. 157B (1985) 295.

[5] V.Kazakov, Mod.Phys.Lett. A4(1989) 2125;

E.Brezin and V.Kazakov, Phys.Lett. B236(1990) 144;

M.Douglas and S.Shenker, Nucl.Phys. B335(1990) 635;

D.Gross and A.Migdal, Phys.Rev.Lett. 64(1990) 127.

[6] M.Douglas, Phys.Lett. B238(1990) 176.

[7] M.Fukuma, H.Kawai and R.Nakayama, Int.J.Mod.Phys. A6 (1991) 1385-1406; Comm.Math.Phys. 143 (1992) 371-403.

[8] A. Marshakov, Theor. Math. Phys. 147 (2006) 777 [Teor. Mat. Fiz. 147 (2006) 399] [arXiv:hep-th/0601214].

[9] M. Goulian and M. Li, Phys. Lett. 66 (1991) 2051.

[10] H. Dorn and H. J. Otto, Nucl. Phys. B 429 (1994) 375 [arXiv:hep-th/9403141];

A. Zamolodchikov and Al. Zamolodchikov, Nucl. Phys. B 477 (1996) 577 [arXiv:hepth/9506136]. 
[11] Al. Zamolodchikov, Int. J. Mod. Phys. A 19S2 (2004) 510 [arXiv:hep-th/0312279].

[12] A. Belavin and Al. Zamolodchikov, in hep-th/0510214.

[13] I. Krichever, Commun. Pure. Appl. Math. 47 (1992) 437, hep-th/9205110.

[14] S. Kharchev, A. Marshakov, A. Mironov and A. Morozov, Mod. Phys. Lett. A8 (1993) 1047, [arXiv:hep-th/9208046].

[15] K. Takasaki and T. Takebe, Rev. Math. Phys. 7 (1995) 743 [arXiv:hep-th/9405096].

[16] S. Kharchev and A. Marshakov, in String Theory, Quantum Gravity and the Unification of the Fundamental Interactions, Proceedings of International Workshop on String Theory, Quantum Gravity and the Unification of Fundamental Interactions, Rome, Italy, 1993, World Scientific; arXiv:hep-th/9210072; Int. J. Mod. Phys. A 10, 1219 (1995) [arXiv:hep-th/9303100].

[17] M. Kontsevich, Func. Analysis \& Apps. 25 (1991) 50; Comm. Math.Phys. 147 (1992) 1

[18] S. Kharchev, A. Marshakov, A. Mironov, A. Morozov and A. Zabrodin, Phys. Lett. B 275 (1992) 311 [arXiv:hep-th/9111037]; Nucl. Phys. B 380 (1992) 181 [arXiv:hepth/9201013].

[19] Al. Zamolodchikov, hep-th/0505063, hep-th/0508044;

Al. Zamolodchikov and Y. Ishimoto, in hep-th/0510214.

[20] V. Kazakov, Phys. Lett. A119 (1986) 140;

D. Boulatov and V. Kazakov, Phys. Lett. B186 (1987) 379.

[21] G. Moore, N. Seiberg and M. Staudacher, Nucl. Phys. B362 (1991) 665-709.

[22] A. Belavin and A. Zamolodchikov, arXiv:0811.0450 [hep-th].

[23] Al. Zamolodchikov, "Thermodynamics of Gravitational Ising Model", 2005, unpublished;

A. Zamolodchikov and Al. Zamolodchikov, arXiv:hep-th/0608196.

[24] V. Kazakov and A. Marshakov, J. Phys. A 36 (2003) 3107 [arXiv:hep-th/0211236].

[25] E.Witten, Nucl.Phys. 340 (1990) 281;

R.Dijkgraaf and E.Witten, Nucl.Phys. 342 (1990) 486.

[26] T. Eguchi and S. K. Yang, Mod. Phys. Lett. A 9 (1994) 2893 [arXiv: hep-th/9407134]; T. Eguchi, K. Hori and S. K. Yang, Int. J. Mod. Phys. A 10 (1995) 4203 [arXiv: hepth/9503017]. 
[27] A. S. Losev, A. Marshakov and N. Nekrasov, "Small instantons, little strings and free fermions," in Ian Kogan memorial volume From fields to strings: circumnavigating theoretical physics, 581-621 [arXiv: hep-th/0302191];

A. Marshakov and N. Nekrasov, JHEP 0701 (2007) 104 [arXiv: hep-th/0612019];

A. Marshakov, Theor. Math. Phys. 154 (2008) 362 arXiv:0706.2857 [hep-th]; JHEP 0803 (2008) 055 [arXiv:0712.2802 [hep-th]]; arXiv:0810.1536.

[28] T. Nakatsu, Y. Noma and K. Takasaki, Int. J. Mod. Phys. A 23 (2008) 2332 [arXiv:0806.3675 [hep-th]]; Nucl. Phys. B 808 (2009) 411 [arXiv:0807.0746 [hep-th]]; T. Nakatsu and K. Takasaki, arXiv:0807.4970. 